\title{
Registros Hospitalares de Câncer de Mato Grosso: análise da completitude e da consistência
}

\section{Hospital-based cancer registries of Mato Grosso, Brazil: analysis of completeness and consistency}

\author{
Jânia Cristiane de Souza Oliveira' (D), Elicleia Filgueira Santiago de Azevedo² (D),

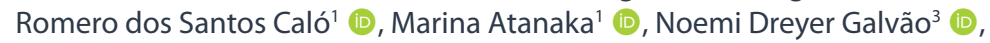 \\ Ageo Mário Cândido da Silva' (D) \\ ${ }^{1}$ Programa de Pós-graduação em Saúde Coletiva, Instituto de Saúde Coletiva, Universidade Federal de Mato Grosso (UFMT) - \\ Cuiabá (MT), Brasil. \\ ${ }^{2}$ Faculdade de Ciências da Saúde, Universidade Federal de Rondonópolis (UFR) - Rondonópolis (MT), Brasil. \\ ${ }^{3}$ Projeto de Extensão, Instituto de Saúde Coletiva, Universidade Federal de Mato Grosso (UFMT) - Cuiabá (MT), Brasil.
}

Como citar: Oliveira JCS, Azevedo EFS, Caló RS, Atanaka M, Galvão ND, Silva AMC. Registros Hospitalares de Câncer de Mato Grosso: análise da completitude e da consistência. Cad Saúde Colet, 2021;29(3):330-343. https://doi. org/10.1590/1414-462X202129030230

\section{Resumo}

Introdução: As informações dos Registros Hospitalares de Câncer (RHC) são fundamentais para a vigilância do câncer. A análise da qualidade dessas informações permite à unidade hospitalar planejar recursos e avaliar a assistência oferecida à pessoa com essa doença. Objetivo: Analisar a qualidade das informações ao verificar a completitude e a consistência das bases de dados do RHC do estado de Mato Grosso (MT). Método: Estudo quantitativo que avaliou os dados dos RHC/MT, de 2008 a 2014. A tendência temporal das proporções de incompletude foi analisada por regressão polinomial e respectiva significância estatística $(p<0,05)$. A análise foi realizada por meio dos softwares Epi Info 7.0 e SPSS 25.0. Resultados: Fizeram parte do estudo um total de 15.090 registros de câncer. As variáveis que apresentaram maior incompletude foram: tumor-nódulometástase, escolaridade, estado da doença ao final do primeiro tratamento e ocupação. Conclusão: Educação permanente visando ao maior envolvimento dos profissionais eà melhoria do registro no prontuário é essencial para o aprimoramento das informações da base hospitalar. Ademais, o uso contínuo dessas informações para a gestão da própria unidade hospitalar subsidia o planejamento e a tomada de decisão, além de avaliar a qualidade da assistência prestada.

Palavras-chave: neoplasias; sistemas de informação; melhoria de qualidade.

\begin{abstract}
Background: Information from the Hospital-based cancer registries (HBCR) is fundamental to watch cancer, analyzing that information allows the Hospital Unit to plan its resources, as well as evaluate the assistance offered to people who suffer from cancer. Objective: To analyze the quality of information by verifying the completeness and consistency of the HBCR from the state of Mato Grosso (MT), Brazil. Method: This is a quantitative study that evaluated the HBCR/MT, from 2008 to 2014. The time trend of the incompleteness proportions was analyzed by polynomial regression and its respective statistical significance $(p<0,05)$. The analysis was done by the software Epi Info 7.0 and SPSS 25.0. Results: A total of 15.090 cancer records were used in the study. The variables that presented the most incompleteness were: tumor-nodule-metastasis; schooling; final state of the disease at the end of the first treatment; and occupation. Conclusion: Permanent Education, which aims for a bigger involvement of health professionals and an improvement of records registration, is essential to enhance information from the hospital database. Also, the continuous use of that information for the own Hospital Unit management funds the planning and the decision making, besides evaluating the quality of the assistance given.
\end{abstract}

Keywords: neoplasms; information systems; quality improvement.

Trabalho realizado no Instituto de Saúde Coletiva, Universidade Federal de Mato Grosso (UFMT) - Cuiabá (MT), Brasil. Correspondência: Jânia Cristiane de Souza Oliveira. E-mail: jania.ufmt@gmail.com

Conflito de interesses: nada a declarar.

Recebido em: Jun. 10, 2019. Aprovado em: Jul. 20, 2020
Este é um artigo publicado em acesso aberto (Open Access) sob a licença Creative Commons Attribution, que permite uso, distribuição e reprodução em qualquer meio, sem restrições desde que o trabalho original seja corretamente citado. 


\section{INTRODUÇÃO}

As doenças crônicas não transmissíveis (DCNT) são a primeira causa de morte mundial e um dos maiores desafios para o século XXI. Em 2016, elas foram responsáveis por 41 milhões de mortes (71\%) no mundo, dos 57 milhões de óbitos que ocorreram naquele ano. A primeira causa de morte foi a doença cardiovascular, com 17,9 milhões de óbitos (44\% do total das DCNT e $31 \%$ de todas as causas de morte), seguida do câncer, com 9 milhões de mortes (22\% das DCNT e $16 \%$ do total de óbitos)'.

Apesar de, atualmente, a doença cardiovascular ser a principal causa de morte por DCNT, estima-se que o câncer assuma o topo dessa lista. Para o século XXI, espera-se que o câncer seja a principal barreira para o aumento da expectativa de vida em todos os países do mundo².

A incidência e a mortalidade por câncer estão crescendo rapidamente no mundo. As explicações são complexas, mas refletem o envelhecimento e o aumento da população, assim como mudanças na prevalência e distribuição dos principais fatores de risco para o câncer ${ }^{3}$, sendo que alguns desses fatores estão associados ao desenvolvimento socioeconômico².

O número de casos novos e de óbitos por câncer no mundo foi estimado em, respectivamente, 18,1 milhões e 9,6 milhões para o ano de $2018^{2}$. Para 2040, poderão sobrevir 29,5 milhões de casos novos e 16,4 milhões de óbitos por câncer ${ }^{4}$.

Para o Brasil, estimam-se 625 mil novos casos de câncer para o triênio 2020-2022, com taxa bruta de 371,11/100.000 homens e 277,11/100.000 mulheres. Para o estado de Mato Grosso, por sua vez, estima-se uma taxa bruta de 252,04/100.000 para homens e de 217,31/100.000 para mulheres ${ }^{5}$.

Além do impacto humano, por meio do aumento da sua incidência e mortalidade, o câncer, atualmente, é um problema de saúde pública mundial e financeiro, já que ele mais as DCNT causarão um impacto econômico global de US\$ 47 trilhões nos próximos 20 anos².

No sentido de se mitigar essa ocorrência, um grande número de pesquisadores constituiu um grupo de vigilância global de sobrevida ao câncer com o intuito de divulgar informações sobre as tendências históricas das notificações de câncer nos diversos países que participaram dessa iniciativa ${ }^{6}$.

No Brasil, a vigilância do câncer é realizada por meio do Programa de Epidemiologia e Vigilância do Câncer e seus fatores de risco (PAV), criado em 1999 pelo Instituto Nacional de Câncer (INCA) ${ }^{7}$, com o objetivo de conhecer o quadro dessa doença no Brasil e de seus fatores de risco. Esse programa é descentralizado para as secretarias estaduais de saúde que são responsáveis por desenvolver ações relacionadas aos registros de câncer por meio da implantação, acompanhamento e aprimoramento dos registros de base hospitalar e populacional ${ }^{8}$.

Em relação aos registros de câncer, os de base populacional (RCBP) coletam dados de uma população específica, enquanto os registros hospitalares (RHC) são centros de coleta, armazenamento, processamento de informações, análise e divulgação das informações obtidas por meio de consulta aos prontuários - de forma sistemática e contínua - de informações de pacientes atendidos em uma unidade hospitalar que prestam assistência oncológica, com diagnóstico confirmado de câncer ${ }^{8,9}$. A partir das informações produzidas em um RHC, podese avaliar a instituição quanto à atenção prestada ao paciente oncológico, subsidiar estudos prognósticos, de sobrevida e análises de tendências temporais dos fenômenos associados à enfermidade. As informações dos registros também são essenciais para a definição de políticas institucionais voltadas ao controle do câncer ${ }^{8}$.

A avaliação da qualidade da informação em registro de câncer é realizada por meio de quatro indicadores principais: comparabilidade, validade, timeliness e completitude dos dados. A comparabilidade é analisada conforme a adesão às normas internacionais de codificação das neoplasias, definição da data de incidência e normas para cânceres múltiplos primários, que permitem a comparação das estatísticas geradas em diferentes populações e através do tempo ${ }^{10-12}$.

A completitude dos dados se refere à extensão de cada câncer incidente estar registrado no banco de dados de câncer. As taxas de incidência e a proporção de sobrevida estarão próximas de seus valores máximos se a inclusão de casos novos ocorrer em tempo hábil 10-12.

A timeliness é realizada para comparar a proporção de casos incidentes com a proporção daqueles registrados como "tardiamente" ${ }^{\prime 10,11}$. 
A validade, ou acurácia, dos dados se refere à proporção de casos registrados com dada característica que realmente possui esse atributo, a depender da qualidade dos documentos utilizados como fonte de dados do registro, bem como da destreza de quem extrai os dados e os codifica ${ }^{10,11}$.

A análise da consistência compõe a validade dos dados dos Registros Hospitalares de Câncer (RHC). Determinado dado não é obrigatoriamente correto apenas por estar presente no banco. Torna-se imperativo verificar se é realmente correto ${ }^{12}$.

São raros os estudos que analisaram a qualidade das informações dos sistemas de informação hospitalar de câncer no Brasil ${ }^{7,13}$.

Assim, este trabalho tem o objetivo de analisar a tendência histórica de completitude e consistência dos registros de câncer da base de dados do RHC no estado de Mato Grosso.

\section{MÉTODO}

Foi realizado estudo descritivo de completitude e validade a partir das bases de dados dos RHC do estado de Mato Grosso disponíveis para download na página eletrônica do Integrador $\mathrm{RHC}(\mathrm{IRHC})$, do INCA.

Os dados são consolidados no Sistema de Registro de Câncer (SisRHC), aplicativo informatizado desenvolvido pelo Ministério da Saúde (MS), por intermédio do INCA, para fortalecer e expandir ainda mais esse sistema integrado de informação sobre câncer. Nessa perspectiva, o INCA desenvolveu um conjunto de ações para padronizar e garantir apoio técnico e divulgar os dados, a partir do IRHC, um sistema informatizado via internet para o envio, a consolidação, o acompanhamento e a análise dos dados nacionais dos RHC brasileiros ${ }^{14}$.

A população do estudo foi constituída pelos casos de câncer que tiveram a primeira consulta na unidade hospitalar no período de 2008 a 2014. A seleção desse período justificase pelo fato de que, a partir de 2008, foi iniciada a inclusão de dados no IRHC das mesmas instituições hospitalares que compõem a base de dados de Mato Grosso, e 2014 foi o último ano com dados atualizados para as seis instituições que compõem os RHC/MT, à época da realização do estudo (Quadro 1). Foram incluídos apenas os casos registrados como analíticos, ou seja, aqueles em que o planejamento da terapêutica do paciente era realizado pelo próprio hospital de registro do câncer, independentemente se alguma etapa do tratamento fosse realizada em outra instituição.

Quadro 1. Hospitais do estado de Mato Grosso, habilitação em oncologia e total de casos analíticos de 2008 a 2014

\begin{tabular}{|c|c|c|c|}
\hline Hospitais & Habilitação & $\begin{array}{c}\text { Total de } \\
\text { casos }\end{array}$ & $\begin{array}{l}\% \text { de } \\
\text { casos }\end{array}$ \\
\hline \multicolumn{4}{|l|}{ RHC Cuiabá } \\
\hline $\begin{array}{l}\text { Hospital de Câncer de Mato Grosso/Associação Mato- } \\
\text { grossense de Combate ao Câncer - AMCC }\end{array}$ & UNACON & 8.214 & 54,43 \\
\hline $\begin{array}{l}\text { Hospital da Sociedade Beneficente Santa Casa de } \\
\text { Misericórdia de Cuiabá }\end{array}$ & UNACON & 3.205 & 21,24 \\
\hline $\begin{array}{l}\text { Hospital Geral Universitário/Associação de Proteção à } \\
\text { Maternidade e à Infância de Cuiabá }\end{array}$ & UNACON & 1.042 & 6,91 \\
\hline Hospital Universitário Júlio Müller & --- & 785 & 5,20 \\
\hline \multicolumn{4}{|l|}{ RHC Interior } \\
\hline Santa Casa de Misericórdia e Maternidade de Rondonópolis & UNACON & 1.439 & 9,54 \\
\hline $\begin{array}{l}\text { Hospital Santo Antônio/Fundação de Saúde Comunitária de } \\
\text { SINOP }\end{array}$ & UNACON & 405 & 2,68 \\
\hline TOTAL & & 15.090 & 100,00 \\
\hline
\end{tabular}

Fonte: Portaria SAS/MS no 102, de 02/03/2012 ${ }^{15}$ 
A qualidade do banco de dados foi avaliada conforme a completitude e a consistência das informações.

Para mensurar a dimensão da completitude, foi realizado o cálculo do percentual de campos incompletos, considerando-se como "campo completo" o registro cujo preenchimento não continha o campo preenchido com o código de informação"ignorado" ou "sem informação".

Nesta análise, foram selecionadas as variáveis classificadas como "obrigatórias" de serem preenchidas na Ficha de Registro de Tumor, a saber: identificação do paciente (sexo, idade, raça/ cor da pele, escolaridade na época da matrícula, ocupação e procedência); caracterização do diagnóstico e do tumor; data do primeiro diagnóstico; diagnóstico e tratamento anteriores; base mais importante para o diagnóstico; topografia; tumor-nódulo-metástase (TNM); estadiamento; caracterização do primeiro tratamento (data do início do primeiro tratamento específico para o tumor no hospital, principal razão para a não realização do tratamento antineoplásico no hospital, primeiro tratamento recebido no hospital e estado da doença ao final do primeiro tratamento). Ainda foram consideradas as variáveis de preenchimento opcional utilizadas para a avaliação do prognóstico, quais sejam: estado conjugal atual; histórico familiar de câncer; histórico de consumo de bebida alcóolica; histórico de consumo de tabaco; lateralidade do tumor.

As variáveis foram analisadas segundo a proporção de casos preenchidos com códigos inexistentes ou formato incorreto. Também foram avaliadas as proporções de casos de inconsistência entre as variáveis: topografia e lateralidade, localização primária desconhecida e localização provável do tumor primário.

Para a avaliação entre a topografia e a lateralidade, foram utilizados como referência os procedimentos de edição entre campos do Surveillance, Epidemiology, and End Results (SEER) para lateralidade ${ }^{16}$, considerando-se os seguintes estratos válidos: direito, esquerdo, bilateral e não se aplica. Foram classificados como inconsistentes os casos com topografia que possuíam lateralidade (por exemplo, rim) e foram registrados como "não se aplica"; e os casos com topografia que não possuíam lateralidade (por exemplo, traqueia) e foram registrados como "direito", "esquerdo" ou "bilateral".

Para localização desconhecida e localização provável do tumor primário, foram utilizados como referências a Classificação Internacional de Doenças para Oncologia (CID-O) e o Manual de Rotinas e Procedimentos dos RHC.

$\mathrm{Na}$ avaliação da completitude e da consistência dos dados, foi empregado o escore proposto por Romero e Cunha (2006) ${ }^{17}$ : excelente (menor que 5\%), bom (5 a 10\%), regular (10 a $20 \%$ ), ruim ( 20 a $50 \%$ ) e muito ruim ( $50 \%$ ou mais).

A análise de tendência da incompletude dos dados foi realizada por meio de modelos de regressão polinomial. Os percentuais de incompletude da série foram considerados como variável dependente $(\mathrm{Y})$, e os anos estudados, como variável independente $(\mathrm{X})$.

Para as variáveis que apresentaram algum grau de incompletude, foram realizados testes para encontrar a curva que melhor descrevesse a relação entre as variáveis dependente e independente. Os modelos de regressão polinomial utilizados estão detalhados a seguir:

- $\quad$ Linear: $y=\beta_{0}+\beta_{1} x$

- Quadrático: $y=\beta_{0}+\beta_{1} x+\beta_{2} x^{2}$

- Cúbico: $y=\beta_{0}+\beta_{1} x+\beta_{2} x^{2}+\beta_{3} x^{3}$

- $\quad$ Exponencial: $y=\beta_{0}{ }^{*} \exp \left(\beta_{1}{ }^{*} \mathrm{x}\right) \beta \leftrightarrow \ln (\mathrm{y})=\left(\beta_{0}\right)+\left(\beta_{1} \mathrm{x}\right)$

Os valores $\mathrm{X}$ e $\mathrm{Y}$ representam as variáveis independente e dependente, respectivamente. $\beta_{0^{\prime}} \beta_{1^{\prime}} \beta_{2}$ e $\beta_{3^{\prime}}$ por sua vez, indicam os coeficientes de regressão polinomial. Foram elaborados 18 modelos de regressão polinomial, cuja variável dependente foi a porcentagem de incompletude dos dados da Ficha de Registro de Tumor dos RHC de Mato Grosso. O modelo foi considerado capaz de descrever a relação entre as variáveis dependente e independente quando o valor de $p$ foi $<0,05$. Para as situações em que mais de um modelo contemplou esse quesito, foi feita a opção pelo que apresentou o menor valor de $p$; e quando o valor de $p$ foi coincidente, foi feito uso do modelo mais simples.

A análise do percentual dos dados foi realizada por meio do programa Epi Info 7.0, e a análise de tendência, com o auxílio do Statistical Package for the Social Sciences (SPSS), versão 25. 


\section{RESULTADOS}

No período estudado, foi registrado um total de 20.299 casos de câncer, e todos foram classificados como analíticos ou não analíticos, informação essencial para o planejamento e o gerenciamento das ações de uma unidade hospitalar que presta atendimento oncológico em Mato Grosso. Foram excluídos 5.209 registros classificados como não analíticos.

Dos 15.090 analisados, 13.246 (87,78\%) registros eram de Cuiabá e 1.844 (12,22\%) eram do interior, sendo $1.996(13,23 \%)$ registrados em 2008, $1.856(12,30 \%)$ em 2009, $1.937(12,84 \%)$ em 2010, 1.966 (13,03\%) em 2011, 2.122 (14,06\%) em 2012, 2.471 em 2013 (16,38\%) e 2.742 (18,17\%) em 2014, apresentando um aumento progressivo com incremento de $27,21 \%$ (Tabela 1).

Tabela 1. Incompletude e inconsistência das variáveis, segundo a proporção de casos sem informação e proporção dos códigos inexistentes, respectivamente, RHC/MT 2008-2014

\begin{tabular}{|c|c|c|c|c|}
\hline \multirow{2}{*}{ Variáveis } & \multicolumn{2}{|c|}{ Incompletude \% } & \multicolumn{2}{|c|}{ Inconsistência \% } \\
\hline & $\begin{array}{c}\text { Cuiabá } \\
\text { (n=13.246) }\end{array}$ & $\begin{array}{c}\text { Interior } \\
(n=1.844)\end{array}$ & $\begin{array}{c}\text { Cuiabá } \\
(n=13.246)\end{array}$ & $\begin{array}{c}\text { Interior } \\
(n=1.844)\end{array}$ \\
\hline \multicolumn{5}{|l|}{ Identificação do paciente } \\
\hline Sexo & 0,00 & 0,00 & $0,00^{*}$ & 0,00 \\
\hline Idade & 0,02 & 0,00 & 0,00 & 0,00 \\
\hline Raça/cor & 15,39 & 35,30 & 0,00 & 0,00 \\
\hline Escolaridade & 57,53 & 87,20 & 0,00 & 0,00 \\
\hline Ocupação & 33,53 & 72,72 & 0,00 & 0,00 \\
\hline Procedência & 1,83 & 57,54 & 0,00 & 0,00 \\
\hline \multicolumn{5}{|l|}{ Caracterização do diagnóstico e do tumor } \\
\hline Data da primeira consulta no hospital & 0,00 & 0,00 & 0,00 & 0,00 \\
\hline Data do primeiro diagnóstico do tumor & 0,40 & 3,74 & 0,00 & 0,00 \\
\hline Diagnóstico e tratamento anteriores & 0,14 & 0,00 & 0,00 & 0,00 \\
\hline Base mais importante para o diagnóstico & 0,20 & 0,05 & 0,00 & 0,00 \\
\hline TNM & 77,22 & 5,69 & 0,01 & 0,00 \\
\hline Estadiamento & 42,17 & 2,01 & 0,00 & 0,00 \\
\hline \multicolumn{5}{|l|}{ Caracterização do primeiro tratamento } \\
\hline Data do início do primeiro tratamento & 2,95 & 7,00 & 0,00 & 0,00 \\
\hline Principal razão para o não tratamento & 14,53 & 17,84 & 0,00 & 0,00 \\
\hline Primeiro tratamento recebido & 3,20 & 1,23 & 0,00 & 0,00 \\
\hline Estado da doença ao final do primeiro tratamento & 46,95 & 24,46 & 0,00 & 0,00 \\
\hline \multicolumn{5}{|l|}{ Opcionais ${ }^{* *}$} \\
\hline Estado conjugal & 11,17 & 71,53 & 0,00 & 7,97 \\
\hline Histórico familiar de câncer & 73,37 & 98,21 & 0,00 & 7,97 \\
\hline Histórico de consumo de bebida alcoólica & 69,06 & 82,59 & 0,00 & 7,97 \\
\hline Histórico de consumo de tabaco & 68,45 & 82,10 & 0,00 & 7,97 \\
\hline Lateralidade & 14,45 & 72,02 & 0,00 & 7,97 \\
\hline
\end{tabular}

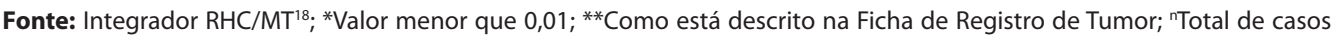
registrados no RHC de Cuiabá e do Interior, conforme Quadro 1 
Em relação à inconsistência de digitação, as informações "estado conjugal atual", "histórico familiar de câncer","histórico de consumo de tabaco","histórico de bebida alcóolica" e"lateralidade" apresentaram a mesma proporção de erro de 7,97\% $(n=147)$, em que, no local das informações corretas constantes do código de registro hospitalar do câncer, constava o código "0", inexistente na ficha. Todos esses erros de digitação ocorreram no ano de 2008 e eram referentes a lançamentos dos municípios do interior. Também foram observadas inconsistências nas variáveis sexo e TNM, ambas no banco de Cuiabá, a primeira com proporção de erro de $0,007 \%$ e a segunda com $0,01 \%$ (Tabela 1 ).

Do total de registros, $6,69 \%(n=1.009)$ apresentaram inconsistência entre as informações "topografia" e "lateralidade do tumor". Entre as topografias que apresentaram lateralidade válida, os que tiveram maior percentual de erro de preenchimento proporcional em relação ao total de sua topografia foram: "glândula parótida" (96,77\%), "amígdala" (84,91\%), "testículo" (53,52\%), "glândula suprarrenal" (50\%) e"ureter" (50\%). Entre as topografias que não possuíam lateralidade, as que tiveram maior percentual de erro foram: "traqueia" (100\%), "timo" (50\%), "intestino delgado" (47,06\%), "vagina" (30,77\%),"gengiva inferior" (25\%) e"glândula paratireoide" (25\%) (Tabela 2).

Tabela 2. Inconsistência entre a topografia e a lateralidade, RHC/MT 2008-2014

\begin{tabular}{|c|c|c|c|}
\hline Topografia codificada como órgão par & $\mathbf{n}$ & $\mathbf{n}^{*}$ & $\%$ \\
\hline Traqueia (C 33) & 1 & 1 & 100,00 \\
\hline Timo (C 37) & 2 & 1 & 50,00 \\
\hline Intestino delgado (C 17) & 17 & 8 & 47,06 \\
\hline Vagina (C 52) & 13 & 4 & 30,77 \\
\hline Gengiva inferior (C 03) & 4 & 1 & 25,00 \\
\hline Glândula paratireoide (C 75) & 4 & 1 & 25,00 \\
\hline Cólon (C 18) & 452 & 84 & 18,58 \\
\hline Fígado (C 22) & 43 & 10 & 23,26 \\
\hline Lábio (C 00) & 20 & 4 & 20,00 \\
\hline Seio piriforme (C 12) & 24 & 4 & 16,67 \\
\hline Estômago (C 16) & 353 & 57 & 16,15 \\
\hline Vesícula biliar (C 23) & 19 & 3 & 15,79 \\
\hline Reto (C 20) & 398 & 38 & 9,55 \\
\hline Placenta (C 58) & 14 & 2 & 14,29 \\
\hline Hipofaringe (C 13) & 32 & 5 & 15,69 \\
\hline Bexiga (C 67) & 161 & 21 & 13,04 \\
\hline Próstata (C 61) & 2.197 & 176 & 8,01 \\
\hline Útero (C 55) & 69 & 6 & 8,69 \\
\hline Esôfago (C 15) & 254 & 25 & 9,84 \\
\hline Pâncreas (C 25) & 72 & 7 & 9,72 \\
\hline Assoalho anterior da boca e assoalho da boca SOE (C 04) & 25 & 2 & 8,00 \\
\hline
\end{tabular}

Fonte: Integrador $\mathrm{RHC} / \mathrm{MT}^{18} . \mathrm{n}=$ total de casos para esta topografia no banco $\mathrm{RHC} / \mathrm{MT}$, para o período analisado; $\mathrm{n}^{*}=$ total de casos inconsistentes para a topografia no banco RHC/MT, para o período analisado; **Treze topografias com percentis menores que 8,00 , SOESem outras especificações 
Tabela 2. Continuação...

\begin{tabular}{lccc}
\multicolumn{1}{c}{ Topografia codificada como órgão par } & $\mathbf{n}$ & n* & $\%$ \\
\hline Outros** $^{*}$ Topografia codificada como órgão único & 2.974 & 88 & 47,97 \\
\hline Glândula parótida (C 07) & 31 & 30 & 96,77 \\
\hline Amígdala (C 09) & 53 & 45 & 84,91 \\
\hline Testículo (C 62) & 71 & 38 & 53,52 \\
\hline Glândula suprarrenal (C 74) & 4 & 2 & 50,00 \\
\hline Ureter (C 66) & 2 & 1 & 50,00 \\
\hline Olho (C 69) & 16 & 7 & 43,75 \\
\hline Ovário (C 56) & 186 & 70 & 37,63 \\
\hline Rim (C 64) & 169 & 58 & 34,32 \\
\hline Mama (C 50) & 2.361 & 210 & 8,89 \\
\hline
\end{tabular}

Fonte: Integrador $\mathrm{RHC} / \mathrm{MT}^{18}$. $\mathrm{n}$ = total de casos para esta topografia no banco $\mathrm{RHC} / \mathrm{MT}$, para o período analisado; $\mathrm{n}^{*}=$ total de casos inconsistentes para a topografia no banco RHC/MT, para o período analisado; ${ }^{* *}$ Treze topografias com percentis menores que 8,00 , SOESem outras especificações

Na análise das séries históricas de completitude, das 21 variáveis analisadas, 3 não foram incluídas nesta análise por não apresentarem incompletude (sexo, idade e data da primeira consulta no hospital). Das variáveis analisadas com campos incompletos no banco RHC/MT, as variáveis "primeiro tratamento recebido no hospital" $e$ "lateralidade" apresentaram tendências crescentes estatisticamente significantes de incompletude dos dados $(p<0,05)$ por meio dos modelos polinomiais cúbico e exponencial, respectivamente. As demais $(88,89 \%)$ possuíram tendências estacionárias $(p>0,05)$. Chama atenção o fato de apenas $1 / 3$ das variáveis apresentar grau de completitude "excelente" ou "bom" e de metade das variáveis apresentar grau de completitude "ruim" ou "muito ruim".

Ao analisar a incompletude do bloco de identificação do paciente, as cidades do interior do estado apresentaram proporção de incompletude maior, comparadas com Cuiabá. É possível notar que houve um melhor preenchimento dos campos referentes ao diagnóstico e ao tratamento dos pacientes, em comparação com os dados sobre informações socioeconômicas. Destaca-se negativamente o registro da variável "escolaridade", que foi classificada como "muito ruim" em todos os bancos. Já a variável "ocupação" foi classificada como de completitude "ruim" no registro de Cuiabá e como "muito ruim" no banco de dados dos municípios do interior de Mato Grosso. "Raça/cor" também foi uma variável avaliada com grau de completitude de registros "regular" no banco de Cuiabá e "ruim" no banco do interior/ MT. Contudo, a variável "procedência" apresentou resultados discrepantes, sendo classificada como de grau de completitude "excelente" no banco de Cuiabá e "muito ruim" no banco do interior/MT (Tabelas 3 e 4).

Das informações de caracterização do diagnóstico e do tumor analisadas, o banco de dados de Cuiabá apresentou menor completitude do que o banco dos municípios do interior do estado. Variáveis como "data do primeiro diagnóstico do tumor", "diagnóstico e tratamento anterior" e "base mais importante para diagnóstico" obtiveram avaliação "excelente" em ambos os bancos. Por outro lado, as variáveis "estadiamento" $e^{\text {"TNM" apresentaram avaliações }}$ diferentes entre os bancos: grau de completitude "excelente" $\mathrm{e}$ "bom" no banco do interior/MT e grau de completitude"ruim"e"muito ruim" no banco de Cuiabá, respectivamente (Tabelas 3 e 4).

Quanto à caracterização do primeiro tratamento, os dois bancos tiveram incompletude semelhante, com percentual discretamente maior do RHC/Cuiabá comparado ao do interior. O banco de Cuiabá possuiu duas variáveis com grau de completitude "excelente", enquanto o do 
Tabela 3. Proporção de incompletude, tendência e grau de completitude dos dados da Ficha de Registro de Câncer, RHC de Cuiabá, Mato Grosso, 2008-2014

\begin{tabular}{|c|c|c|c|c|c|c|}
\hline \multirow[b]{2}{*}{ Variáveis } & \multicolumn{2}{|c|}{ 2008-2014 } & \multicolumn{4}{|c|}{ Tendência } \\
\hline & $n=13.246 \%$ & $\begin{array}{c}\text { Grau de } \\
\text { completitude }\end{array}$ & & $\mathbf{r}^{2(\mathbf{a})}$ & $\mathbf{p}$ & Modelo \\
\hline \multicolumn{7}{|l|}{ Identificação do paciente } \\
\hline Raça/cor & 15,39 & Regular & Estacionário & 0,071 & 0,564 & Exponencial \\
\hline Escolaridade & 57,53 & Muito ruim & Estacionário & 0,005 & 0,881 & Exponencial \\
\hline Ocupação & 33,53 & Ruim & Estacionário & 0,283 & 0,219 & Linear \\
\hline Procedência & 1,83 & Excelente & Estacionário & 0,780 & 0,544 & Exponencial \\
\hline \multicolumn{7}{|l|}{ Caracterização do diagnóstico e do tumor } \\
\hline Data do primeiro diagnóstico do tumor & 0,40 & Excelente & Estacionário & 0,164 & 0,368 & Linear I \\
\hline Diagnóstico e tratamento anteriores & 0,14 & Excelente & Estacionário & 0,625 & 0,342 & Cúbico \\
\hline Base mais importante para o diagnóstico & 0,20 & Excelente & Estacionário & 0,455 & 0,557 & Cúbico \\
\hline (Classificação de tumores malignos) TNM & 77,22 & Muito ruim & Estacionário & 0,687 & 0,098 & Quadrático \\
\hline Estadiamento & 42,17 & Ruim & Estacionário & 0,542 & 0,061 & Exponencial \\
\hline \multicolumn{7}{|l|}{ Caracterização do primeiro tratamento } \\
\hline Data do início do primeiro tratamento & 2,95 & Excelente & Estacionário & 0,062 & 0,591 & Logarítmico \\
\hline Principal razão para o não tratamento & 14,53 & Regular & Estacionário & 0,115 & 0,456 & Linear \\
\hline Primeiro tratamento recebido no hospital & 3,20 & Excelente & Crescente & 0,927 & 0,033 & Cúbico \\
\hline $\begin{array}{l}\text { Estado da doença ao final do primeiro } \\
\text { tratamento }\end{array}$ & 46,95 & Ruim & Estacionário & 0,789 & 0,154 & Cúbico \\
\hline \multicolumn{7}{|l|}{ Opcionais } \\
\hline Estado conjugal atual & 11,17 & Regular & Estacionário & 0,390 & 0,134 & Exponencial \\
\hline Histórico familiar de câncer & 73,37 & Muito ruim & Estacionário & 0,664 & 0,296 & Cúbico \\
\hline Histórico de consumo de bebida alcoólica & 69,06 & Muito ruim & Estacionário & 0,679 & 0,277 & Cúbico \\
\hline Histórico do consumo de tabaco & 68,45 & Muito ruim & Estacionário & 0,683 & 0,272 & Cúbico \\
\hline Lateralidade & 14,45 & Regular & Crescente & 0,941 & 0,024 & Cúbico \\
\hline
\end{tabular}

Fonte: Integrador $\mathrm{RHC} / \mathrm{MT}^{18}$

(a) $r^{2}=$ coeficiente de explicação; " Total de casos registrados no RHC de Cuiabá e do Interior, conforme Quadro 1. p<0,05

interior possuiu somente uma variável. Apenas a variável "estado da doença ao final do primeiro tratamento" foi avaliada com grau de completitude "ruim" em ambos os bancos (Tabelas 3 e 4).

Das informações consideradas como de preenchimento opcional para avaliação de prognóstico da doença, o banco do interior apresentou menor completitude nos registros em relação ao banco da capital. Enquanto o banco de Cuiabá possuiu grau de completitude "regular" para as variáveis "estado conjugal" e "lateralidade" e "muito ruim" para as demais, o banco do interior/MT apresentou grau de completitude "muito ruim" para todas as variáveis desse grupo (Tabelas 3 e 4).

\section{DISCUSSÃO}

O resultado do estudo mostrou as limitações nos bancos dos RHC de Cuiabá e do interior do estado de Mato Grosso para o período do estudo. Foi observada baixa completitude para variáveis de grande relevância para a descrição do perfil de casos de câncer. Apesar de 33,33\% 
Tabela 4. Proporção de incompletude, tendência e grau de completitude dos dados da Ficha de Registro de Câncer, RHC do interior de Mato Grosso, 2008-2014

\begin{tabular}{|c|c|c|c|c|c|c|}
\hline \multirow{2}{*}{ Variáveis } & \multicolumn{2}{|c|}{ 2008-2014 } & \multicolumn{4}{|c|}{ Tendência } \\
\hline & $n=1.844 \%$ & $\begin{array}{c}\text { Grau de } \\
\text { completitude }\end{array}$ & & $\mathbf{r}^{2(a)}$ & $\mathbf{p}$ & Modelo \\
\hline \multicolumn{7}{|l|}{ Identificação do paciente } \\
\hline Raça/cor & 35,30 & Ruim & Estacionário & 0,700 & 0,090 & Quadrático I \\
\hline Escolaridade & 87,20 & Muito ruim & Crescente & 0,944 & 0,022 & Cúbico \\
\hline Ocupação & 72,72 & Muito ruim & Crescente & 0,977 & 0,006 & Cúbico \\
\hline Procedência & 57,54 & Muito ruim & Crescente & 0,970 & $<0,001$ & Linear \\
\hline \multicolumn{7}{|l|}{ Caracterização do diagnóstico e do tumor } \\
\hline Data do primeiro diagnóstico do tumor & 3,74 & Excelente & Estacionário & 0,328 & 0,451 & Quadrático \\
\hline Diagnóstico e tratamento anteriores & 0,00 & Excelente & Estacionário & 0,000 & 0,000 & --- \\
\hline Base mais importante para o diagnóstico & 0,05 & Excelente & Estacionário & 0,064 & 0,585 & Logarítmico \\
\hline $\begin{array}{l}\text { (Classificação de tumores malignos) } \\
\text { TNM }\end{array}$ & 5,69 & Bom & Decrescente & 0,614 & 0,037 & Linear \\
\hline Estadiamento & 2,01 & Excelente & Decrescente & 0,867 & 0,002 & Logarítmico \\
\hline \multicolumn{7}{|l|}{ Caracterização do primeiro tratamento } \\
\hline Data do início do primeiro tratamento & 7,00 & Bom & Estacionário & 0,699 & 0,091 & Quadrático \\
\hline Principal razão para o não tratamento & 17,84 & Regular & Estacionário & 0,457 & 0,095 & Linear \\
\hline Primeiro tratamento recebido no hospital & 1,23 & Excelente & Estacionário & 0,202 & 0,312 & Linear \\
\hline $\begin{array}{l}\text { Estado da doença ao final do primeiro } \\
\text { tratamento }\end{array}$ & 24,46 & Ruim & Estacionário & 0,782 & 0,161 & Cúbico \\
\hline \multicolumn{7}{|l|}{ Opcionais } \\
\hline Histórico familiar de câncer & 71,53 & Muito ruim & Crescente & 0,851 & 0,022 & Quadrático \\
\hline Estado conjugal atual & 98,21 & Muito ruim & Crescente & 0,987 & $<0,001$ & Quadrático \\
\hline $\begin{array}{l}\text { Histórico de consumo de bebida } \\
\text { alcoólica }\end{array}$ & 82,59 & Muito ruim & Crescente & 0,996 & $<0,001$ & Cúbico \\
\hline Histórico do consumo de tabaco & 82,10 & Muito ruim & Crescente & 0,996 & $<0,001$ & Cúbico \\
\hline Lateralidade & 72,02 & Muito ruim & Crescente & 0,989 & $<0,001$ & Quadrático \\
\hline
\end{tabular}

Fonte: Integrador $\mathrm{RHC} / \mathrm{MT}^{18}$

(a) $\mathrm{r}^{2}$ = coeficiente de explicação; ${ }^{\mathrm{n}}$ Total de casos registrados no RHC de Cuiabá e do Interior, conforme Quadro 1. p<0,05

das variáveis dos $\mathrm{RHC} /$ Cuiabá e 38,89\% das variáveis do banco do interior apresentarem grau de completitude "excelente" ou "bom", as que tiveram grau de completitude "ruim" ou "muito ruim" foram superiores, com $44,44 \%$ e $55,56 \%$, respectivamente. Além disso, 10 variáveis, sendo $8(44,44 \%)$ no banco do interior e $2(11,11 \%)$ no banco de Cuiabá, possuíram tendências crescentes estatisticamente significantes de incompletude dos dados.

O déficit de informação pode refletir a fragilidade de preenchimento dos prontuários de pacientes atendidos nessas instituições, a falta de prontuário único, o discreto envolvimento dos profissionais de saúde no preenchimento correto, completo e claro dos documentos institucionais dos pacientes ${ }^{19}$ ou até mesmo a letra inelegível dos prontuários médicos ${ }^{20,21}$. A análise dessas variáveis permite à instituição incremento de suas ações, facilitando o planejamento dos recursos e a tomada de decisão.

O excelente grau de preenchimento para as variáveis sexo, idade e data da primeira consulta reflete que dados mínimos de informação, como sexo, data de nascimento e 
primeira consulta realizada na instituição, constam no prontuário da pessoa atendida na unidade hospitalar. Resultado similar foi observado no estudo de Pinto et al. ${ }^{13}$, com grau de completitude excelente para as variáveis sexo e idade. Talvez esse achado seja decorrente da baixa subjetividade de interpretação necessária para o registro dessas informações.

Neste estudo, a variável escolaridade apresentou um dos piores escores, corroborando pesquisas realizadas no $\mathrm{Brasi}^{7,13}$ que evidenciaram incompletude de $69 \%$ e de $31,2 \%$ para essa variável. $O$ estudo da variável escolaridade é considerado relevante, pois pode ser indicativo de desigualdade social, além de sua utilidade como proxy de fator socioeconômico, quando não se tem acesso à informação da renda ${ }^{22,23}$. $O$ estudo dessa variável permite ainda realizar comparações, como busca precoce por diagnóstico, adesão ao tratamento, avaliação de sobrevida e recidiva da doença, entre outras. Várias pesquisas foram realizadas na busca de analisar os fatores associados à escolaridade e ao câncer de mama ${ }^{24-26}$. Esses estudos apontam que, talvez por causa do estilo de vida e comportamento, o resultado das mulheres com escolaridade maior foi melhor que das demais.

Outra variável que foi avaliada com grau de completitude "ruim" e "muito ruim" foi a ocupação. Moraes et al. ${ }^{27}$ descreveram o perfil ocupacional de indivíduos com leucemia, cuja completitude dos dados foi de $52 \%$. Ainda nessa pesquisa, foi observado elevado número de sub-registros de dados, além de não conter no RHC informações quanto ao princípio ativo utilizado, tempo de exposição ao longo de sua vida laboral e dados de ocupações prévias. Há vários estudos que associam determinadas ocupações com uma chance maior de desenvolver câncer ou mesmo ir a óbito por essa causa, o que enfatiza a necessidade de maior detalhamento no registro das informações relativas à atividade de trabalho ${ }^{28-31}$.

Para a variável raça/cor, neste estudo foi constatado grau de completitude "regular" e "ruim", resultado similar encontrado em pesquisas realizadas no Brasil',13. O estudo da raça/ cor possui relevância singular, pois pode refletir não apenas uma diferença biológica, mas também desigualdade de acesso a diagnóstico e tratamento de câncer. Estudos realizados nos Estados Unidos com mulheres com câncer de mama demonstraram resultado estatisticamente significativo na disparidade dos óbitos pela doença em mulheres negras, se comparado às demais ${ }^{32-34}$. Outros estudos, ao analisarem a sobrevida de mulheres com câncer de mama, evidenciaram que as mulheres negras apresentaram sobrevida inferior, mesmo após ajuste para fatores socioeconômicos e status hormonal ${ }^{35,36}$. Apesar da diferença da biologia tumoral, carga genética e cuidados com a saúde, talvez o fator que mais contribua para essa disparidade racial sejam as iniquidades ${ }^{34}$.

Grau de completitude "muito ruim" e "ruim" foi verificado para as variáveis TNM e estadiamento, respectivamente, no banco da capital, Cuiabá. Estudos realizados no Brasi ${ }^{7,13}$ encontraram grau de completitude "ruim" para ambas variáveis, ao analisarem dados do RHC de Minas Gerais e do Brasil, respectivamente.

Conhecer oTNM e o estadiamento é imprescindível, pois permite distinguir a extensão da doença no momento do diagnóstico. A partir dessa informação, é definido o plano terapêutico adotado e, dessa maneira, é possível avaliar o resultado do tratamento administrado à pessoa com câncer, o que facilita a padronização de procedimentos e troca de experiências entre instituições que oferecem tratamento oncológico. Conhecer o estadiamento também contribui para a avaliação da assistência oferecida à pessoa com câncer, além de subsidiar a implantação e a implementação de políticas de diagnóstico precoce ${ }^{9,37}$. A relevância dessas informações se deve ainda por causa de sua relevância enquanto fator prognóstico, muito utilizado em estudos de sobrevida.

Aspectos relacionados ao tratamento subsidiam a avaliação da assistência recebida pela pessoa com câncer e são fundamentais para seu plano terapêutico. Pesquisas realizadas com dados do RHC do Brasil e de Minas Gerais observaram grau de completitude "regular" para a variável estado da doença ao final do primeiro tratamento. Vários estudos foram realizados ${ }^{25,38-42}$ com o objetivo de analisar diferentes tipos de tratamento. Para comparar os procedimentos realizados na assistência oncológica, informações acerca do tratamento precisam estar registradas no prontuário. 
A variável procedência foi avaliada como "muito ruim" no banco do interior. Estudo realizado na Austrália evidenciou que mulheres com câncer de mama não localizado que residiam em área rural possuíam sobrevida menor que as mulheres com o mesmo tipo de câncer que residiam em área urbana ${ }^{43}$. Para que seja possível analisar a procedência como potencial fator de risco, proteção ou prognóstico, é necessária a completitude da informação dessa variável. Vale ressaltar também a associação entre ambiente e câncer, já comprovada em vários trabalhos ${ }^{28,44,45}$.

Neste estudo, as variáveis de preenchimento opcional foram classificadas com grau de completitude baixo. Vale destacar a relevância dessas variáveis para conhecer o perfil da pessoa com câncer e realizar estudos de prognóstico. Além disso, várias pesquisas já associaram vários desses itens (estado conjugal, consumo de tabaco, consumo de álcool, história familiar da doença, lateralidade) com o câncer ${ }^{46-50}$. Seria interessante incorporar tais variáveis com as de preenchimento obrigatório.

As variáveis consumo de tabaco e consumo de bebida alcoólica foram classificadas com grau de completitude "muito ruim" em ambos os bancos. Estudo de D'Alessandro et al.7 evidenciou grau de completitude "ruim" para essas duas variáveis. Outras pesquisas já comprovaram a associação entre o consumo de álcool e tabaco e o câncer de boca e orofaringe ${ }^{47,48,50}$. O consumo de tabaco isolado também está associado a doenças coronarianas, câncer de pulmão e doença obstrutiva crônica ${ }^{49}$.

A variável estado conjugal atual foi classificada como"regular" no banco de Cuiabá e"muito ruim" no banco do interior, inferior aos resultados de D'Alessandro et al.?. Estudo realizado nos Estados Unidos ${ }^{46}$ evidenciou que mulheres com câncer de mama e que possuíam relações sociais apresentavam níveis de inflamação menores que as que não possuíam. Já a pesquisa de Martinez et al. ${ }^{51}$ evidenciou que mulheres com câncer de mama e solteiras tinham maior mortalidade por todas as causas em comparação com as casadas, porém esse benefício de sobrevida variava de acordo com padrões raciais/étnicos, grupos, por subtipo do tumor e status socioeconômico da vizinhança.

O preenchimento da variável lateralidade, neste estudo, apresentou grau de completitude "regular" e "muito ruim". Resultado similar ao achado dos RHC/Cuiabá foi encontrado em D'Alessandro et al. ${ }^{7}$, com grau de completitude "regular" para essa variável. Pesquisa realizada por Bao et al. ${ }^{49}$ demonstrou o resultado da análise por meio da anotação completa da localização do tumor e evidenciou que os tumores localizados na região inferior interna da mama, em ambos os lados, tendiam a apresentar pior prognóstico que nas demais localizações.

Em relação à consistência dos dados, foi observado percentil elevado de inconsistência entre topografia e lateralidade, se comparado ao número total da topografia contida no banco. Talvez isso ocorra por causa da nomenclatura entre a topografia da CID-O e a classificação da lateralidade. Por exemplo, uma topografia que apresentou dados inconsistentes foi a referente ao cólon (C 18), registrada como cólon ascendente (cólon direito C 18.2) e cólon descendente (cólon esquerdo $C 18.6)^{52}$, porém quanto à lateralidade é órgão único ${ }^{16}$. Vale ressaltar que o registrador não é um profissional da área da saúde, o que emerge a necessidade de educação permanente sobre a temática.

Este estudo apresenta limitações próprias de pesquisa com base de dados secundários, entretanto traz contribuições no sentido de abordar um tema que cada vez mais ocupa relevância epidemiológica no cenário brasileiro e mundial, além de apontar lacunas no registo do câncer no sistema de informação hospitalar.

Os problemas encontrados na base dos RHC/MT sinalizam a necessidade de realização de educação permanente não apenas para os registradores, mas também para a equipe multiprofissional que presta atendimento à pessoa com câncer para anotação da informação correta, completa e clara no prontuário. Ademais, a base dos RHC é extremamente rica e útil para fornecer subsídios para o planejamento e a avaliação da unidade hospitalar, com a finalidade de contribuir para a qualidade da vigilância do câncer, desde o diagnóstico precoce, o tratamento e os cuidados adequados aos pacientes oncológicos. 


\section{REFERÊNCIAS}

1. World Health Organization. World health statistics 2019: monitoring health for the SDGs, sustainable development goals [Internet]. Geneva:WHO; 2019 [citado em 2020 Abr 20]. Disponível em: https://www. who.int/data/gho/publications

2. Bray F, Ferlay J, Soerjomataram I, Siegel RL, Torre LA, Jemal A. Global cancer statistics 2018: GLOBOCAN estimates of incidence mortality worldwide for 36 cancers in 185 countries. CA Cancer J Clin. 2018;68(6):394424. http://dx.doi.org/10.3322/caac.21492. PMid:30207593.

3. Omran AR. The epidemiologic transition: a theory of the epidemiology of population change. Bull World Health Organ. 2001;79(2):161-70. PMid:11246833.

4. World Health Organization. International Agency for Cancer Research. Global Cancer Observatory. Cancer tomorrow [Internet]. Geneva: WHO; 2020 [citado em 2020 Abr 20]. Disponível em: https://gco.iarc.fr/ tomorrow/home

5. Instituto Nacional de Câncer José Alencar Gomes da Silva. Coordenação de Prevenção e Vigilância. Estimativa 2020: incidência de câncer no Brasil. Rio de Janeiro: INCA; 2019.

6. Allemani C, Matsuda T, Di Carlo V, Harewood R, Matz M, Nikšić M, et al. Global surveillance of trends in cancer survival 2000-14 (CONCORD-3): analysis of individual records for 37513025 patients diagnosed with one of 18 cancers from 322 population-based registries in 71 countries. Lancet. 2018 mar;391(10125):1023-75. http://dx.doi.org/10.1016/S0140-6736(17)33326-3. PMid:29395269.

7. D'Alessandro TAL, Antoniazzi BN, Abreu DMX. Registros Hospitalares de Câncer de Minas Gerais: análise de consistência das bases de dados. Cad Saude Colet. 2010;18(3):410-7.

8. Instituto Nacional de Câncer José Alencar Gomes da Silva. Coordenação Geral de Prevenção e Vigilância, Divisão de Vigilância e Análise da Situação. Manual de rotinas e procedimentos para registros de câncer de base populacional. 2. ed. Rio de Janeiro: INCA; 2012.

9. Instituto Nacional de Câncer José Alencar Gomes da Silva. Coordenação de Prevenção e Vigilância. Secretaria Nacional de Assistência à Saúde. Registros hospitalares de câncer: planejamento e gestão. 2. ed. Rio de Janeiro: INCA; 2010. 538 p.

10. Sigurdardottir LG, Jonasson JG, Stefansdottir S, Jonsdottir A, Olafsdottir GH, Olafsdottir EJ, et al. Data quality at the Icelandic Cancer Registry: Comparability, validity, timeliness and completeness. Acta Oncol. 2012;51(7):880-9. http://dx.doi.org/10.3109/0284186X.2012.698751. PMid:22974093.

11. Wanner M, Matthes KL, Korol D, Dehler S, Rohrmann S. Indicators of data quality at the cancer Registry Zurich and Zug in Switzerland. BioMed Res Int. 2018;7656197. http://dx.doi.org/10.1155/2018/7656197. PMid:30009174.

12. Skeet RG. Qualidade e controle de qualidade. In: Jensen OM, Parkin DM, MacLennan R, Muir CS, Skeet RG, editors. Registros de câncer: princípios e métodos. Rio de Janeiro: Coordenadoria de Programas de Controle do Câncer/INCA; 1995. p. 107-13.

13. Pinto IV, Ramos DN, Costa MCE, Ferreira CBT, Rebelo MS. Completude e consistência dos dados dos registros hospitalares de câncer no Brasil. Cad Saude Colet. 2012;20(1):113-20.

14. Instituto Nacional de Câncer José Alencar Gomes da Silva. Coordenação Geral de Prevenção e Vigilância. Registro hospitalar de câncer: planejamento e gestão. Rio de Janeiro: INCA; 2017.

15. Ministério da Saúde. Secretaria de Atenção à Saúde. Portaria SAS/MS no 102, de 03 de fevereiro de 2012 Habilitações de Serviços Especializados do Sistema do Cadastro Nacional de Estabelecimentos de Saúde.

16. Van Holten V. Edição para consistência de dados. In: Jensen OM, Parkin DM, MacLennan R, Muir CS, Skeet RG, editores. Registros de câncer: princípios e métodos. Rio de Janeiro: Coordenadoria de Programas de Controle do Câncer/INCA; 1995. Apêndice 2.

17. Romero $\mathrm{DE}, \mathrm{Cunha} \mathrm{CB}$. Avaliação da qualidade das variáveis sócio-econômicas e demográficas dos óbitos de crianças menores de um ano registrados no Sistema de Informações sobre Mortalidade do Brasil (1996/2001). Cad Saude Publica. 2006;22(3):673-84. http://dx.doi.org/10.1590/S0102-311X2006000300022. PMid:16583111.

18. Instituto Nacional de Câncer. Integrador RHC - Registro Hospitalar de Câncer. Disponível em: https://irhc. inca.gov.br/RHCNet/visualizaTabNetExterno.action

19. Silveira DP. Perfil da incidência e da sobrevida do câncer de mama: análise a partir dos Registros de Câncer de Base Populacional e cobertura de planos privados de saúde no município de São Paulo [tese]. Rio de Janeiro: Escola Nacional de Saúde Pública, Fiocruz; 2011.

20. Sampaio AC, Silva MRF. Prontuário médicos: reflexo das relações médico-paciente. Rev Bioet. 2010;18(2):451-68. 
21. Sampaio AC. Qualidade dos prontuários médicos como reflexos das relações médico-usuário em cinco hospitais de Recife/PE [tese]. Recife: Centro de Pesquisas Aggeu Magalhaes, Fiocruz Pernambuco; 2010.

22. Soares AM Fo, Souza MFM, Gazal-Carvalho C, Malta DC, Alencar AP, Silva MMA, et al. Análise da mortalidade por homicídios no Brasil. Epidemiol Serv Saude. 2007;16(1):7-18.

23. Rebelo PAP, Lima RGM, Souto Rebelo M. Registros hospitalares de câncer - rotinas e procedimentos. Rio de Janeiro: INCA; 2000.

24. Davies H, Marcu A, Vedsted P, Whitaker KL. Is lower symptom recognition associated with socioeconomic inequalities in help-seeking for potencial breast cancer symptoms? Psychooncology. 2018;27(2):626-32. http://dx.doi.org/10.1002/pon.4557. PMid:28940942.

25. Diniz RW, Guerra MR, Cintra JRD, Fayer VA, Teixeira MTB. Disease-free survival in patients with nonmetastatic breast cancer. Rev Assoc Med Bras. 2016;62(5):407-13. http://dx.doi.org/10.1590/18069282.62.05.407. PMid:27656849.

26. Gadgil A, Roy N, Sankaranarayanan R, Muwonge R, Sauvaget C. Effect of comprehensive breast care on breast cancer outcomes: a community Hospital Based Study from Mumbai, India. Asian Pac J Cancer Prev. 2012;13(4):1105-9. http://dx.doi.org/10.7314/APJCP.2012.13.4.1105. PMid:22799289.

27. Moraes ES, Mello MSC, Nogueira FAM, Otero UB, Carvalho FN. Análise de indivíduos com leucemia: limitações do sistema de vigilância de câncer. Cien Saude Colet. 2017;22(10):3321-32. http://dx.doi. org/10.1590/1413-812320172210.18292017. PMid:29069187.

28. Pignati W, Oliveira NP, da Silva AM. Vigilância aos agrotóxicos: quantificação do uso e previsão de impactos na saúde-trabalho-ambiente para os municípios brasileiros. Cien Saude Colet. 2014;19(12):4669-78. http:// dx.doi.org/10.1590/1413-812320141912.12762014. PMid:25388175.

29. Miranda-Filho AL, Monteiro GTR, Meyer A. Brain cancer mortality among farm workers of the State of Rio de Janeiro, Brazil: a population-based case-control study, 1996-2005. Int J Hyg Environ Health. 2012;215(5):496-501. http://dx.doi.org/10.1016/j.ijheh.2011.10.007. PMid:22118878.

30. Bezerra AG, Souza AT, Carvalho PRL, Bedor CNG. Identificação do potencial carcinogênico dos agrotóxicos: tetraconazol, ciproconazol, triadimenol, poxadiazona e cloransulam-metílico [Internet]. Evolvere Scientia. 2013;2(1):9-18. [citado em 2018 Dez 30]. Disponível em: http://www.scientia.univasf.edu.br/vol2/paper02pp9-18.pdf

31. Viero CM, Camponogara S, Cezar-Vaz MR, Costa VZ, Beck CLC. Sociedade de risco: o uso dos agrotóxicos e implicações na saúde do trabalho rural. Esc Anna Nery. 2016;20(1):99-105. http://dx.doi.org/10.5935/14148145.20160014.

32. Sighoko D, Hunt BR, Irizarry B, Watson K, Ansell D, Murphy AM. Dispatiry in breast cancer mortality by age and geography in 10 racially diverse US cities. Cancer Epidemiol. 2018;53:178-83. http://dx.doi. org/10.1016/j.canep.2018.02.003. PMid:29477058.

33. Eng LG, Dawood S, Sopik V, Haaland B, Tan PS, Bhoo-Pathy N, et al. Ten-year survival in women with primary stage IV breast cancer. Breast Cancer Res Treat. 2016;160(1):145-52. http://dx.doi.org/10.1007/ s10549-016-3974-x. PMid:27628191.

34. Hunt BR, Hurlbert MS. Black:White disparities in breast cancer mortality in the 50 largest cities in the United States, 2005-2004. Cancer Epidemiol. 2016;45:169-73. http://dx.doi.org/10.1016/j.canep.2016.07.018. PMid:27720130.

35. Agarwal S, Ying J, Boucher KM, Agarwal JP. The association between socioeconomic factors and breast cancer-specific survival varies by race. PLoS One. 2017;12(12):e0187018. http://dx.doi.org/10.1371/journal. pone.0187018. PMid:29211739.

36. DeSantis CE, Ma J, Goding Sauer A, Newman LA, Jemal A. Breast cancer statistics, 2017, racial disparity in mortality by State. CA Cancer J Clin. 2017;67(6):439-48. http://dx.doi.org/10.3322/caac.21412. PMid:28972651.

37. Instituto Nacional de Câncer José Alencar Gomes da Silva. Coordenação Geral de Prevenção e Vigilância. TNM: classificação de tumores malignos. 7. ed. Rio de Janeiro: INCA; 2012. 325 p.

38. Houvenaeghel G, Boher JM, Michel V, Bannier M, Minsat M, Tallet A, et al. Survival after breast cancer local recurrence according to therapeutic strategies. EJSO. 2017;43(8):1409-14. http://dx.doi.org/10.1016/j. ejso.2017.04.006. PMid:28536053.

39. Nagar H, Yan W, Christos P, Chao C, Nori D, Ravi A. Older patients with early-stage breast cancer adjuvant radiation therapy and predictive factors for cancer-related death. Am J Clin Oncol. 2017;40(3):300-5. http://dx.doi.org/10.1097/COC.0000000000000144. PMid:25333731. 
40. Barinoff J, Schmidt M, Schneeweiss A, Schoenegg W, Thill M, Keitel S, et al. Primary metastatic breast cancer in the era of targeted therapy - Prognostic impact and the role of breast tumor surgery. Eur $J$ Cancer. 2017;83:116-24. http://dx.doi.org/10.1016/j.ejca.2017.06.002. PMid:28735068.

41. Sundquist M, Brudin L, Tejler G. Improved survival in metastatic breast cancer 1985-2016. Breast. 2017;31:46-50. http://dx.doi.org/10.1016/j.breast.2016.10.005. PMid:27810699.

42. Bello MA, Menezes RF, Silva B, da Silva RC, Cavalcanti RS, Moraes TD, et al. Impact of treatment type on overall survival in elderly Brazilian women with breast cancer. Asian Pac J Cancer Prev. 2016;17(10):476974. http://dx.doi.org/10.22034/APJCP.2016.17.10.4769. PMid:27893210.

43. Yu XQ, Luo Q, Kahn C, O'Connell DL, Houssami N. Temporal trends show improved breast cancer survival in Australia but widening urban - rural differences. Breast. 2015;24(4):524-7. http://dx.doi.org/10.1016/j. breast.2015.03.006. PMid:25843905.

44. Nasrala E No, Lacaz FAC, Pignati WA. Vigilância em saúde e agronegócio: os impactos dos agrotóxicos na saúde e no ambiente. Perigo à vista! Cien Saude Colet. 2014 dez;19(12):4709-18. http://dx.doi. org/10.1590/1413-812320141912.03172013. PMid:25388179.

45. Curvo HRM, Pignati WA, Pignatti MG. Morbimortalidade por câncer infatojuvenil associada ao uso Agrícola de agrotóxicos no Estados de Mato Grosso, Brasil. Cad Saude Colet. 2013;21(1):10-7. http://dx.doi. org/10.1590/S1414-462X2013000100003.

46. Busch EL, Whitsel EA, Kroenke $\mathrm{CH}$, Yang YC. Social relationships, inflammation markers, and breast cancer incidence in the Women's Health Initiative. Breast. 2018;39:63-9. http://dx.doi.org/10.1016/j. breast.2018.03.013. PMid:29614476

47. Amorim FS Fo, Andrade Sobrinho J, Rapoport A, Carvalho MB, Novo NF, Juliano Y. Estudo de variáveis demográficas, ocupacionais e co-carcinogenéticas no carcinoma espinocelular da base de língua nas mulheres. Rev Bras Otorrinolaringol. 2003;69(4):472-8. http://dx.doi.org/10.1590/S003472992003000400006.

48. Brasil. Ministério da Saúde. Secretaria de Atenção à Saúde. Departamento de Atenção Básica. Coordenação Nacional de Saúde Bucal. Normas e Manuais Técnicos. Brasília: Ministério da Saúde; 2008. 92 p. (Série A. Cadernos de Atenção Básica; no. 17).

49. Bao J, Yu KD, Jiang YZ, Shao ZM, Di GH. The effect of laterality and primary tumor site on cancer-specific mortality in breast cancer: a SEER population-based study. PLoS One. 2014;9(4):e94815. http://dx.doi. org/10.1371/journal.pone.0094815. PMid:24740002.

50. Instituto Nacional de Câncer José Alencar Gomes da Silva. Coordenação de Prevenção e Vigilância. Secretaria Nacional de Assistência à Saúde. A situação do câncer no Brasil. Rio de Janeiro: INCA; 2006. $120 \mathrm{p}$.

51. Martinez ME, Unkart JT, Tao L, Kroenke CH, Schwab R, Komenaka I, et al. Prognostic significance of marital status in breast cancer survival: a population-based study. PLoS One. 2017;12(5):e0175515. http://dx.doi. org/10.1371/journal.pone.0175515. PMid:28475579.

52. Organização Mundial de Saúde. CID-0 - Classificação Internacional de Doenças para Oncologia. São Paulo: Editora da Universidade de São Paulo; 2005. 